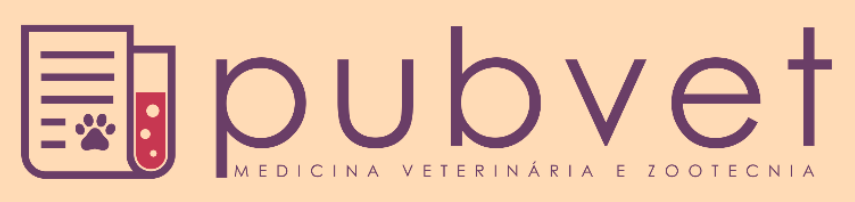

https://doi.org/10.31533/pubvet.v14n2a520.1-7

\title{
Diagnostico de cisticercose bovina em frigorífico na região noroeste do estado de São Paulo, Brasil
}

\author{
Yane Caroline Horas do Nascimento ${ }^{1 *} \bullet$, Paulo Rogério Dobre ${ }^{2} \bullet$, Affonso dos Santos \\ $\operatorname{Marcos}^{3} \bullet$, Rosângela Felipe Rodrigues ${ }^{4}{ }^{\bullet}$, Alan Peres Ferraz de Melo ${ }^{4} \bullet$ \\ ${ }^{1}$ Faculdade de Engenharia, Graduanda em Zootecnia/UNESP. \\ ${ }^{2}$ Faculdade de Engenharia, Curso de Pós-graduação em Ciência e Tecnologia Animal/UNESP. \\ ${ }^{3}$ UNESP-FEIS, Assistente Agropecuário. Coordenadoria de Defesa Agropecuária e Abastecimento. Governo do Estado de São Paulo. \\ ${ }^{4}$ Faculdade de Medicina Veterinária de Araçatuba/UNESP. \\ *Autor para correspondência, E-mail: vanechn@gmail.com
}

Resumo. A cisticercose bovina é uma zoonose causada pela Taenia Saginata, que oferece grande risco a população consumidora de carne bovina e com grande impacto na produção animal. O trabalho teve como objetivo fazer um diagnóstico da prevalência da cisticercose bovina na região Noroeste do estado de São Paulo, Brasil e investigar quais são os municípios que mais fornecem animais para o abate. O diagnóstico da zoonose foi feito utilizando o método de visualização de cistos na carcaça em inspeção realizada sob o Serviço de Inspeção Oficial, com a supervisão de um Médico Veterinário. Para a estimativa da prevalência foram utilizados dados do período do ano de 2007 ao ano de 2016 fornecidos pelo Frigorifico Ilha Solteira Ltda. e para a investigação das cidades de origem dos animais foram utilizados dados do ano de 2007 ao ano de 2014. A prevalência de cisticercose bovina na região Noroeste do Estado de São Paulo, Brasil entre os anos de 2007 a 2016 foi de 4,83\%, e os municípios que mais forneceram animais para abate no Frigorifico Ilha Solteira Ltda. foram Pereira Barreto, seguido pelos municípios de Santo Antônio do Araranguá, Sud Mennucci, Mirandópolis e Guaraçaí.

Palavras chave: cisticercose, bovino, frigorífico, região Noroeste

\section{Diagnosis of bovine cysticercosis in refrigerators in the northwestern region of the state of São Paulo, Brazil}

Abstract. Bovine cysticercosis is a zoonosis caused by Taenia Saginata, which presents a high risk to the consuming population of bovine meat and with great impact on animal production. The objective of this study was to diagnose the prevalence of bovine cysticercosis in the Northwest region of the state of São Paulo, Brazil, and to investigate which municipalities provide the most animals for slaughter. The diagnosis of zoonosis was made using the method of visualization of cysts in the carcass in inspection performed under the Official Inspection Service, under the supervision of a Veterinarian. For the estimation of the prevalence data were used from the period of the year 2007 to the year 2016 provided by Frigorific Ilha Solteira Ltda. and for the investigation of the cities of origin of the animals were used data from the year 2007 to the year 2014. The prevalence of bovine cysticercosis in the Northwest region of the State of São Paulo, Brazil between the years 2007 to 2016 was $4.83 \%$, and the municipalities that provided the most animals for slaughter at Frigorific Ilha Solteira Ltda. were Pereira Barreto, followed by the municipalities of Santo Antônio do Araranguá, Sud Mennucci, Mirandópolis and Guaraçai.

Keywords: Cysticercosis, bovine, Frigorific, Northwest region 


\section{Diagnóstico de cisticercosis bovina en planta de sacrificio en la región noroeste del estado de São Paulo, Brasil}

Resumen. La cisticercosis bovina es una zoonosis causada por Taenia Saginata, que presenta un alto riesgo para la población consumidora de carne bovina y tiene un gran impacto en la producción animal. El objetivo de este estudio fue diagnosticar la prevalencia de cisticercosis bovina en la región noroeste del estado de São Paulo, Brasil, e investigar qué municipios proporcionan la mayor cantidad de animales para el sacrificio. El diagnóstico de zoonosis se realizó utilizando el método de visualización de quistes en la canal en una inspección realizada bajo el Servicio de Inspección Oficial, bajo la supervisión de un médico veterinario. Para la estimación de prevalencia se utilizaron los datos desde el período del año 2007 hasta el año 2016 proporcionados por el frigorífico Ilha Solteira Ltda. y para la investigación de las ciudades de origen de los animales se utilizaron datos desde el año 2007 hasta el año 2014. La prevalencia de cisticercosis bovina en la región noroeste del estado de São Paulo, Brasil entre los años 2007 a 2016 fue de $4.83 \%$ y los municipios que proporcionaron la mayor cantidad de animales para el sacrificio en la planta Ilha Solteira Ltda. fueron Pereira Barreto, seguidos por los municipios de Santo Antônio do Araranguá, Sud Mennucci, Mirandópolis y Guaraçai.

Palabras clave: Cisticercosis, bovina, frigorífica, región noroeste

\section{Introdução}

A cisticercose bovina é uma zoonose de alcance mundial, sendo considerada uma doença de países subdesenvolvidos por ser comumente encontrada em regiões mais pobres e com populações pouco informadas sobre a doença (OMS, 1994). Essa doença pode oferecer grande risco a consumidores da carne contaminada. Além de apresentar grandes riscos à população, a cisticercose bovina é uma zoonose de grande impacto econômico na cadeia produtiva da carne. Por ser a zoonose mais comum em frigoríficos é considerada a principal causadora de condenação de carcaça de bovinos abatidos em frigoríficos sob Inspeção Veterinária Oficial (Khaniki et al., 2010; Marques et al., 2008; Vieira et al., 2011), pois pode causar perda de partes da carcaça, gerando danos financeiros ao produtor, sem contar que impacta na economia de países produtores de carne, pois gera uma limitação nas exportações do produto.

Essa doença faz parte do ciclo do complexo teníase-cisticercose e se caracteriza como o estágio larval do parasita Taenia saginata. Sua ocorrência é caracterizada como um problema de saúde pública, sendo causada por problemas sanitários recorrentes em propriedades de criação de animais, assim como hábitos de consumo errôneos da carne, como o mau cozimento (Pfuetzenreiter \& Pires, 2000; Scandrett et al., 2009). Entre as ações que estão associadas à prevalência da zoonose na produção de bovinos se encontra o sistema de criação de animais em locais contaminados e o abate na própria propriedade, onde não há inspeção sanitária dos animais abatidos (Almeida et al., 2002; Flisser et al., 2003; Gomes et al., 2008).

Observando o cenário mundial, percebe-se prevalência muito baixa em países desenvolvidos da Europa, como mostrado em estudos nas regiões da França (Dupuy et al., 2014), Espanha (Allepuz et al., 2009) e Dinamarca (Calvo-Artavia et al., 2013) em contraste com regiões do mundo mais pobres como a América Latina, continente Africano e Ásia (Giri \& Parija, 2012; Phiri et al., 2003) em que a prevalência se mostra bastante alta. No Brasil a média nacional é considerada de $5 \%$ (Souza et al., 2007), portanto um país endêmico, segundo a Organização Pan-Americana de Saúde (OPAS, 1994).

Além da prevalência, conhecer a origem dos animais é muito importante para que o foco do problema seja identificado e estratégias sejam implementadas por órgãos de saúde pública. Dados geográficos ambientais são cada vez mais analisados na investigação da cisticercose bovina (AcevedoNieto, 2015; Dutra et al., 2012), sendo cada vez mais desenvolvidos os estudos na área de sistema de informação geográfica (SIG), que mapeia rebanhos e locais infectados (Acevedo-Nieto et al., 2012; 
Bavia et al., 2012; Guimarães-Peixoto et al., 2012). Esse mapeamento permite a melhor visualização da doença, tornando mais facil a organização de planos de prevenção e de estratégias de controle.

Considerando esses fatores o presente trabalho teve como objetivo investigar a prevalência de cisticercose na região Noroeste do estado de São Paulo entre os anos de 2007 a 2016 e as cidades de origem desses animais abatidos no Frigorífico Ilha Solteira Ltda. entre os anos de 2007 a 2014.

\section{Material e métodos}

Foram utilizados registros de inspeção de abate de 275.580 bovinos fêmeas e machos. Os animais tiveram origem de diferentes municípios localizados na região Noroeste do estado de São Paulo, Brasil. Os dados analisados dos bovinos abatidos foram entre o período de janeiro de 2007 a dezembro de 2016 no Frigorifico Ilha Solteira Ltda. que se encontra sob Serviço de Inspeção Federal (SIF) e está localizado no município de Ilha Solteira, estado de São Paulo, Brasil.

O frigorífico executa sua inspeção sob supervisão do Serviço de Inspeção oficial, seguindo todas as normas brasileiras impostas para a inspeção post mortem de carcaças e vísceras, com o devido acompanhamento de um médico-veterinário. A rotina de inspeção consiste na visualização de lesões em partes específicas da carcaça, como musculatura esquelética, coração, músculos de mastigação, língua e diafragma. Em casos que cistos eram identificados a carcaça era analisada pelo médicoveterinário, onde o cisto era classificado como cisto calcificado ou cisto vivo.

Para a estimativa da prevalência foram organizados em planilhas a quantidade anual de animais abatidos, assim como o número de cistos vivos e cistos calcificados encontrados nas carcaças. A prevalência obtida consistiu na média de cistos encontrados entre os anos de 2007 a 2016.

Para a investigação dos municípios de origem dos animais recebidos para abate, também foram utilizados dados fornecidos pelo frigorífico em que foram organizados em tabelas a quantidade de animais que cada município forneceu no período do ano de 2007 ao ano de 2014. Dos municípios analisados foram destacados os que mais forneceram animais nesse período.

\section{Resultados}

Das 275.580 carcaças inspecionadas entre os anos de 2007 a 2016, foram verificados 940 cistos vivos e 11.491 cistos calcificados, resultando em uma média de prevalência de 4,83\% (Tabela 1).

A menor média anual encontrada foi no ano de 2014 e 2015 em que teve uma prevalência de 2,2\%, sendo a maior média anual encontrada no ano de 2009, com prevalência de 8,6\%. Entre os anos a maior diminuição da doença foi do ano de 2012 a 2013 em que a prevalência foi de 4,70\% para 2,94\%, resultando em uma queda de $62,5 \%$ na prevalência (Gráfico 1). Os cistos calcificados foram encontrados em maior quantidade, correspondendo a $92,44 \%$ do total de cistos.

Tabela 1. Prevalência de cisticercose em carcaças de bovinos abatidos no Frigorífico Ilha Solteira Ltda. entre os anos de 2007 a 2016

\begin{tabular}{lcccc}
\hline Ano & Animais abatidos & Cisto vivo & Cisto calcificado & Prevalência \\
\hline 2007 & 27.271 & 186 & 1.962 & $7,70 \%$ \\
2008 & 20.061 & 151 & 1.642 & $8,40 \%$ \\
2009 & 17.712 & 145 & 1.370 & $8,60 \%$ \\
2010 & 22.942 & 122 & 1.070 & $5,20 \%$ \\
2011 & 26.657 & 108 & 1.043 & $4,30 \%$ \\
2012 & 36.678 & 133 & 1.369 & $4,70 \%$ \\
2013 & 33.140 & 64 & 909 & $2,94 \%$ \\
2014 & 33.083 & 16 & 709 & $2,20 \%$ \\
2015 & 30.015 & 8 & 653 & $2,20 \%$ \\
2016 & 28.021 & 7 & 764 & $2,78 \%$ \\
Total & 275.580 & 940 & 11.491 & $4,83 \%$ \\
\hline
\end{tabular}


Nos dados de 2007 a 2014 em que foram analisados os municípios de origem dos animais foi verificado o recebimento de animais de cem municípios da região Noroeste de São Paulo, Brasil entre esses anos e verificou-se maior recebimento de animais para abate dos municípios de Pereira Barreto, seguido pelos municípios de Santo Antônio do Aracanguá, Sud Mennucci, Mirandópolis e Guaraçaí, que somaram 46,62\% dos animais fornecidos para abate entre 2007 a 2014 (Tabela 2).

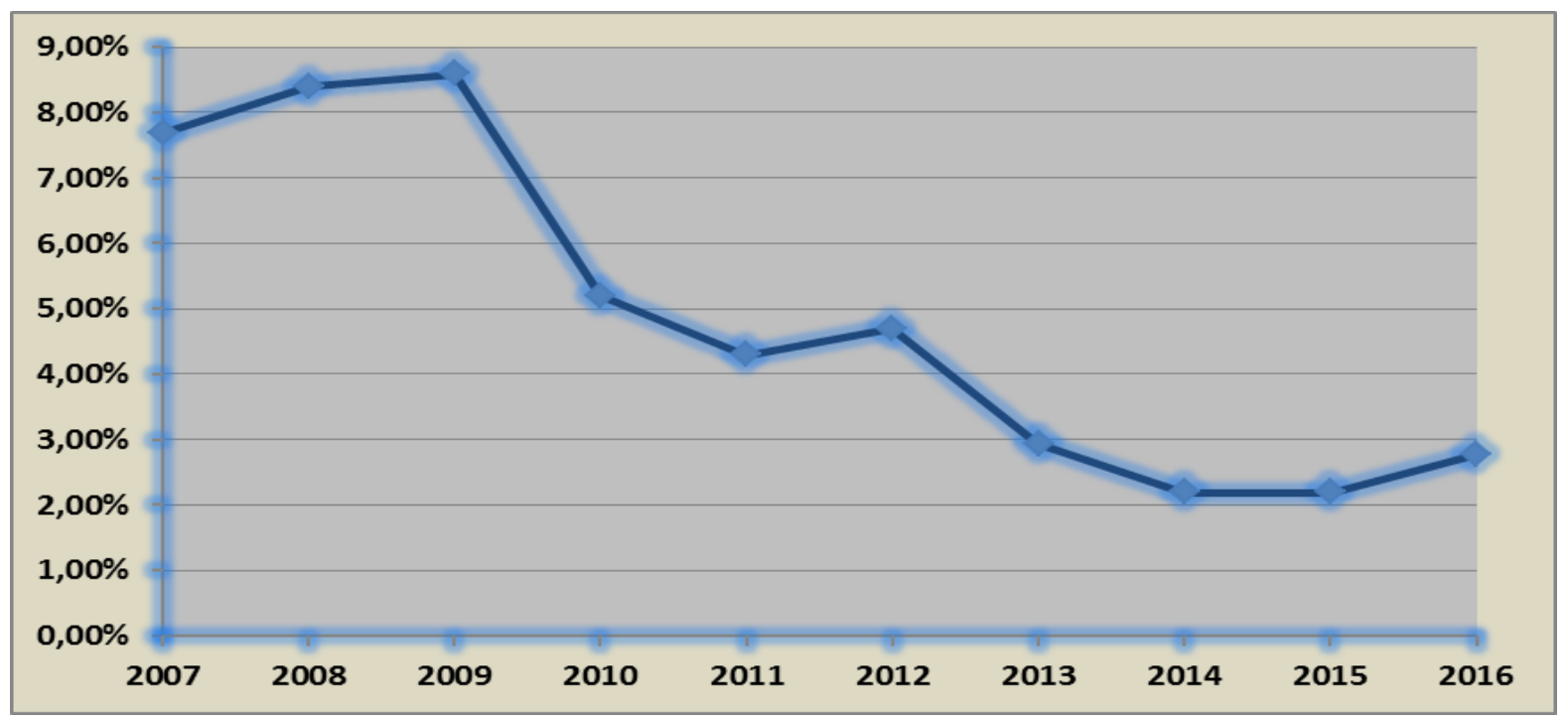

Gráfico 1. Decréscimo da prevalência da cisticercose na região noroeste do Estado de São Paulo entre os anos de 2007 a 2016.

Tabela 2. Cidades que mais forneceram animais para abate no Frigorifico Ilha Solteira Ltda. entre os anos de 2007 a 2014.

\begin{tabular}{lccccc}
\hline Pereira Barreto & Sud Mennucci & Sto. Antônio Aracanguá & Mirandópolis & Guaraçaí & Total de animais \\
\hline $23,42 \%$ & $20,76 \%$ & $20,57 \%$ & $19,77 \%$ & $15,48 \%$ & $46,62 \%$ \\
\hline
\end{tabular}

\section{Discussão}

A média de prevalência encontrada se assemelha à média do Estado de São Paulo, Brasil de 4,8\% (Ferreira et al., 2014; Mendes et al., 2005) e do Estado de Minas Gerais, Brasil de 4,7\% (Duarte et al., 2016; Magalhães et al., 2017). Contudo, ainda se manifesta abaixo da média nacional de 5\% (Souza et al., 2007). Segundo indicadores da Organização Pan-Americana de Saúde (OPAS, 1994), apenas são considerados endêmicas regiões que apresentam médias acima de 5\% de prevalência (OPAS, 1994), no entanto, regiões que apresentam infestação acima de 1\% ainda necessitam de vigilância preventiva e controle pela ação de órgãos públicos (Aragão et al., 2017).

No que se refere ao tipo de cisto, foi verificado maior quantidade de cistos calcificados, equivalendo a $92,44 \%$. Os dados que apontam predominância de cistos calcificados na carcaça corroboram com resultados de inspeção relatados por diversos outros autores, 83,50\% (Santos et al., 2001), 64,12\% (Almeida et al., 2002) e 75,46\% (Lima et al., 2011). Essa predominância pode ser atribuída ao fato de que os produtores podem estar vermifugando os animais antes do abate para que ocorra a calcificação do cisto (Lima et al., 2011), havendo também a possibilidade de que os animais estejam sendo abatidos com idade após o tempo de vida do parasito, ocorrendo assim uma calcificação natural.

No decorrer dos anos, foi observada uma expressiva diminuição da cisticercose (Gráfico 1), sendo constatado uma grande queda da prevalência entre o ano de 2012 a 2013. Essa diminuição pode ser atribuída a possível adoção de medidas profiláticas como o cozimento correto da carne, que promove a quebra do ciclo da Taenia, e uma melhora nos hábitos sanitários em propriedades de criação animal, uma vez que água e comidas contaminadas por fezes já se mostraram como a maior via de transmissão da doença (Duarte et al., 2016). Essas atitudes também podem ser relacionadas com o aumento do grau de conhecimento da população sobre a zoonose, que se mostra bastante baixo em regiões de maior infestação (Arrais-Silva et al., 2017; Bürger et al., 2015; Lima et al., 2011). Outra estratégia possivelmente adotada foi a diminuição de trocas de animais entre propriedades, evitando que a 
doença se espalhe e mantendo a prevalência baixa, assim como mostrado no Estado de Mato Grosso do Sul, onde ocorreu o decréscimo de prevalência de $25 \%$ no ano de 2010, para 1,8\% no ano de 2012 utilizando essa estratégia (Aragão et al., 2017).

$\mathrm{Na}$ análise dos dados dos anos de 2007 a 2014 foi verificado o recebimento de animais de cem municípios pertencentes à região Noroeste do Estado de São Paulo, sendo que os maiores fornecedores foram Pereira Barreto, Santo Antônio do Aracanguá, Sud Mennucci, Mirandópolis e Guaraçaí. Em um trabalho realizado entre os anos de 1990 a 2000 foi identificada endemicidade maior que a média nacional no município de Santo Antônio do Aracanguá (5,79\%), sendo bastante superior aos outros municípios, como Pereira Barreto (3,64\%), Mirandópolis (3,88\%) e Sud Mennucci $(4,41 \%)$. Considerando os dados, observa-se que esses municípios fazem fronteira entre si, formando uma região muito importante para investigação de um possível foco e causa da doença. A investigação das propriedades fornecedoras desses animais é essencial para o planejamento e implementação de estratégias para a diminuição da prevalência, que impacta não apenas a região em questão, mas também toda a região de distribuição da carne, que nesse caso é bastante extensa.

\section{Conclusão}

A prevalência de cisticercose bovina na região Noroeste do Estado de São Paulo, Brasil foi de $4,83 \%$. Apesar de ter ocorrido diminuição da doença nos últimos anos ainda é importante que se mantenha medidas preventivas e a vigilância sobre a zoonose.

Como os municípios de Pereira Barreto, Santo Antônio do Aracanguá, Sud Mennucci, Mirandópolis e Guaraçaí foram os maiores fornecedores de animais para abate nos anos de 2007 a 2014, recomenda-se que as propriedades de origem dos animais nesses municípios sejam investigadas para que se obtenha maior rastreabilidade da doença nessa região.

\section{Referências bibliográficas}

Acevedo-Nieto, C. E. (2015). Complexo teníase-cisticercose em assentamentos da Reforma Agrária do estado de Minas Gerais, Brasil. Departamento de Veterinária. Master of Universidade Federal de Viçosa.

Acevedo-Nieto, E. C., Vieira, F. C., Pinto, P. S. P., Silva, L. F., Santos, T. O. \& Gumimarães Peixoto, R. P. M. (2012). Análise de fatores de risco para a infecção de cisticercose bovina: estudo de caso controle a partir de animais abatidos. Semina: Ciências Agrárias, 33(6), 2359-2366.

Allepuz, A., Napp, S., Picado, A., Alba, A., Panades, J., Domingo, M. \& Casal, J. (2009). Descriptive and spatial epidemiology of bovine cysticercosis in North-Eastern Spain (Catalonia). Veterinary Parasitology, 159(1), 43-48.

Almeida, L. P., Moreira, M. D., Reis, D. O., \& Santos, W. L. M. (2002). Cisticercose bovina: um estudo comparativo entre animais abatidos em frigoríficos com serviço de inspeçäo municipal. Higigiene Alimentar, 16(99), 51-55.

Aragão, S. C., Ito, P. K. R. K., Paulan, S. C., Utsunomyia, Y. T., Grisi Filho, J. H. H., \& Nunes, C. M. (2017). Animal movement network analysis as a tool to map farms serving as contamination source in cattle cysticercosis. Pesquisa Veterinária Brasileira, 37(4), 319-324.

Arrais-Silva, W. W., Santos, T. B. A., Aguiar, K. M., Sturmer, M., Feitosa, M. T. N., Lunardi, R. R., \& Siqueira, M. F. C. (2017). Análise do conhecimento de alunos do ensino médio público sobre parasitoses endêmicas na região do Médio Araguaia mato-grossense. Revista Ciência Em Extensão, 13(1), 83-90.

Bavia, M. E., Carneiro, D. D. M. T., Cardim, L. L., Silva, M. M. N., \& Martins, M. S. (2012). Estatística espacial de varredura na detecção de áreas de risco para a cisticercose bovina no estado da Bahia. Arquivo Brasileiro de Medicina Veterinária e Zootecnia, 64(5), 1200-1208.

Bürger, K. P., Buzza, P. L. T., Neto, E. B., dos Santos, L. R., Rossi, G. A. M., \& Martins, A. M. C. V. (2015). Complexo teniose-cisticercose: ocorrência em abatedouro de bovinos e conhecimento de estudantes do ensino médio e consumidores no Estado de São Paulo, Brasil. Revista Brasileira de Ciência Veterinária, 22(1), 1-10. 
Calvo-Artavia, F. F., Nielsen, L. R., Dahl, J., Clausen, D. M., \& Alban, L. (2013). Occurrence and factors associated with bovine cysticercosis recorded in cattle at meat inspection in Denmark in 2004-2011. Preventive Veterinary Medicine, 110(2), 177-182.

Duarte, C. T. D., Pinto, P. S. A., Silva, L. F., Santos, T. O., Acevedo-Nieto, E. C., \& Almeida, L. P. (2016). Perfil da transmissão e prevalência da cisticercose bovina em propriedades rurais do Triângulo Mineiro. Pesquisa Veterinária Brasileira, 36(9), 793-797.

Dupuy, C., Morlot, C., Gilot-Fromont, E., Mas, M., Grandmontagne, C., Gilli-Dunoyer, P., Gay, E., \& Callait-Cardinal, M.-P. (2014). Prevalence of Taenia saginata cysticercosis in French cattle in 2010. Veterinary Parasitology, 203(1-2), 65-72.

Dutra, L. H., Girotto, A., Vieira, R. F. C., Vieira, T. S. W. J., Zangirolamo, A. F., Marquês, F. A. C., Headley, S. A., \& Vidotto, O. (2012). A prevalência e epidemiologia espacial da cisticercose em bovinos abatidos no Brasil. Semina: Ciências Agrárias, 33(5), 1887-1896.

Ferreira, M. M., Revoredo, T. B., Ragazzi, J. P., Soares, V. E., Ferraldo, A. S., Mendonça, R. P. de, \& Lopes, W. D. Z. (2014). Prevalência, distribuição espacial e fatores de risco para cisticercose bovina no estado de São Paulo. Pesquisa Veterinária Brasileira, 34(12), 1181-1185.

Flisser, A., Sarti, E., Lightowlers, M., \& Schantz, P. (2003). Neurocysticercosis: regional status, epidemiology, impact and control measures in the Americas. Acta Tropicaropica, 87(1), 43-51.

Giri, S., \& Parija, S. C. (2012). A review on diagnostic and preventive aspects of cystic echinococcosis and human cysticercosis. Tropical Parasitology, 2(2), 99.

Gomes, A. P., Nunes, E. R., Felippe, K. C., Carneiro, M. C., \& Santos, S. S. (2008). Teníase e cisticercose: breve revisão dos aspectos gerais. Pedíatria, 44(4), 151-156.

Guimarães-Peixoto, R. P. M., Souza, V. K., Pinto, P. S. A., \& Santos, T. O. (2012). Distribuição e identificação das regiões de risco para a cisticercose bovina no Estado do Paraná. Pesquisa Veterinária Brasileira, 32(10), 975-979.

Khaniki, G. R. J., Raei, M., Kia, E. B., Haghi, A. M., \& Selseleh, M. (2010). Prevalence of bovine cysticercosis in slaughtered cattle in Iran. Tropical Animal Health and Production, 42(2), 141-143.

Lima, R. S., França, E. L., Honorio-França, A. C., \& Ferrari, C. K. B. (2011). Prevalência de cisticercose bovina e conhecimento sobre a doença em 20 municípios do estado do Mato Grosso. Revista Panorâmica Online, 12(1), 46-60.

Magalhães, F. C., Santos, T. M., Assis, D. C., Ornellas, C. D., Pinto, P. A., \& Santos, W. M. (2017). Diagnóstico e fatores de risco do complexo teníase-cisticercose bovina no município de Salinas, Minas Gerais. Pesquisa Veterinária Brasileira, 37(3), 205-209.

Marques, G. M., Buzi, K. A., Galindo, L. A., Baldini, E. D., \& Biondi, G. F. (2008). Avaliação dos registros de condenação por cisticercose em bovinos abatidos em frigoríficos da região centro oeste do estado de São Paulo (1996-2000). Revista Veterinária e Zootecnia, 15(1), 114-120.

Mendes, E. C., Silva, S. S., Fonseca, E. A. L. T., Souza, H. R. R., \& Carvalho, R. W. (2005). A neurocisticercose humana na baixada fluminense, estado do Rio de Janeiro, Brasil. Arquivos de Neuro-Psiquiatria, 63(4), 1058-1062.

OMS. Organização Mundial da Saúde, 1994. Classificação Estatística Internacional de Doenças e Problemas Relacionados à Saúde. Décima Revisão, Vol. 2. São Paulo: Centro Colaborador da OMS para Classificação de Doenças em Português, Universidade de São Paulo.

OPS - Organización Panamericana de la Salud. Epidemiologia y control da la teniasis/cisticercosis en América Latina. New York: OPS, 1994.

Pfuetzenreiter, M. R., \& Pires, F. D. Á. (2000). Epidemiology of teniasis/cysticercosis by Taenia solium and Taenia saginata. Ciência Rural, 30(3), 541-548.

Phiri, I. K., Ngowi, H., Afonso, S., Matenga, E., Boa, M., Mukaratirwa, S., Githigia, S., Saimo, M., Sikasunge, C., \& Maingi, N. (2003). The emergence of Taenia solium cysticercosis in Eastern and Southern Africa as a serious agricultural problem and public health risk. Acta Tropica, 87(1), 1323.

Santos, I. F., Mano, S. B., Tortelly, R., Santos, M. L. S., \& Silva, D. A. S. (2001). Estudo da localizaçäo do Cysticercus bovis em coraçöes de bovinos abatidos sob inspeçäo. Higiene 
Alimentar, 15(89), 37-44.

Scandrett, B., Parker, S., Forbes, L., Gajadhar, A., Dekumyoy, P., Waikagul, J., \& Haines, D. (2009). Distribution of Taenia saginata cysticerci in tissues of experimentally infected cattle. Veterinary Parasitology, 164(2-4), 223-231. https://doi.org/http://dx.doi.org/10.1016/j.vetpar.2009.05.015

Souza, V. K., Pessôa-Silva, M. C., Minozzo, J. C., \& Thomaz-Soccol, V. (2007). Prevalência da cistice rcose bovina no estado do Paraná, sul do Brasil: avaliação de 26.465 bovinos inspecionados no SIF 1710. Semina: Ciências Agrárias, 28(4), 675-683.

Vieira, N. P., Faria, P. B., Mattos, M. R., \& Pereira, A. A. (2011). Condenação de fígados bovinos na região sul do estado do Espírito Santo. Arquivo Brasileiro de Medicina Veterinária e Zootecnia, 63(6), 1605-1608.

Recebido: 16 de outubro, 2019

Aprovado: 22 de novembro, 2019

Publicado: 25 de março, 2020.

Licenciamento: Este artigo é publicado na modalidade Acesso Aberto sob a licença Creative Commons Atribuição 4.0 (CC-BY 4.0), a qual permite uso irrestrito, distribuição, reprodução em qualquer meio, desde que o autor e a fonte sejam devidamente creditados 